Jurnal Matematika UNAND

Vol. VIII No. 2 Hal. $93-100$

Edisi Agustus 2019

ISSN : 2303-291X

(C)Jurusan Matematika FMIPA UNAND

\title{
SIFAT-SIFAT K-ALJABAR
}

MEZA APRILISA, NOVA NOLIZA BAKAR, YANITA

Program Studi S1 Matematika,

Fakultas Matematika dan Ilmu Pengetahuan Alam, Universitas Andalas,

Kampus UNAND Limau Manis Padang, Indonesia.

email :meza9869@gmail.com

Diterima 22 Juni 2019 Direvisi 6 Juli 2019 Dipublikasikan 4 Agustus 2019

\begin{abstract}
Abstrak. Pada struktur aljabar, yang biasa dikenal adalah grup dan ring. Selain grup dan ring, juga terdapat struktur aljabar yang disebut K-aljabar. K-Aljabar merupakan suatu struktur aljabar yang diperkenalkan oleh K.H. Dar dan M.Akram pada tahun 2006. Dari suatu K-Aljabar, dapat dibentuk satu atau lebih himpunan bagian yang juga memiliki sifat K-Aljabar terhadap operasi biner yang sama yang kemudian himpunan bagian tersebut dapat dinamakan K-Subaljabar. Sebagaimana halnya pada grup yang terdapat konsep homomorfisma grup, pada K-Aljabar juga terdapat konsep homomorfisma yang dinamakan K-Homomorfisma. Pada tulisan ini dibahas mengenai sifat-sifat yang terkait dengan K-Aljabar, K-Subaljabar dan K-Homomorfisma.
\end{abstract}

Kata Kunci: K-Aljabar, K-Subaljabar, K-Homomorfisma, grup, subgrup, homomorfisma

\section{Pendahuluan}

Struktur aljabar yang biasa dikenal adalah struktur aljabar dengan satu operasi biner, yaitu grup dan struktur aljabar dengan dua operasi biner, yaitu ring. Struktur aljabar selain grup dan ring, juga terdapat struktur aljabar yang disebut K-aljabar.

Gagasan mengenai K-Aljabar pertama kali diperkenalkan oleh K.H. Dar dan M.Akram pada tahun 2006. K-Aljabar merupakan suatu struktur aljabar yang dibangun atas suatu grup $\mathrm{G}$, dengan operasi biner $\odot$, yang didefinisikan sebagai $x \odot y=x * y^{-1}=x y^{-1}$ untuk semua $x, y \in G$. K-Aljabar dinotasikan dengan $(G, *, \odot, e)[1]$. Pada K-Aljabar dikenal adanya sifat-sifat K-Subaljabar dan K-Homomorfisma. Pada tulisan ini akan dibahas sifat-sifat yang berkaitan dengan K-Aljabar, K-Subaljabar, dan K-Homomorfisma.

\section{K-Aljabar}

\subsection{K-Aljabar}

Definisi 2.1. [1] Misalkan $(G, *)$ merupakan suatu grup dengan e suatu unsur identitas di $G$ dan didefinisikan operasi $\odot$ sedemikian sehingga untuk setiap $x, y \in G$, $x \odot y=x * y^{-1}$ maka akan membentuk struktur aljabar baru yaitu $(G, *, \odot, e)$. 
Suatu $(G, *, \odot, e)$ dinamakan $K$-Aljabar, dan untuk setiap $x, y, z \in G$ memenuhi aksioma-aksioma berikut:

(1) $(x \odot y) \odot(x \odot z)=(x \odot((e \odot z) \odot(e \odot y))) \odot x$,

(2) $x \odot(x \odot y)=(x \odot(e \odot y)) \odot x$,

(3) $x \odot x=e$,

(4) $x \odot e=x$,

(5) $e \odot x=x^{-1}$.

Jika grup G adalah grup abelian maka Aksioma 1 dan 2 menjadi:

(1) $(x \odot y) \odot(x \odot z)=(z \odot y)$,

(2) $x \odot(x \odot y)=y$, untuk setiap $x, y, z \in G$.

Contoh 2.2. Misalkan $(\mathbb{R}-\{0\}, \cdot)$ adalah suatu grup dengan identitas $e=1$. Didefinisikan operasi $\odot$ pada $\mathbb{R}-\{0\}$, sehingga $x \odot y=x \cdot y^{-1}=x y^{-1}$, untuk setiap $x, y \in \mathbb{R}-\{0\}$. Akan ditunjukkan bahwa $(\mathbb{R}-\{0\}, \cdot, \odot, 1)$ adalah suatu K-Aljabar. Ambil $x, y, z \in \mathbb{R}-\{0\}$, perhatikan bahwa:

(1) $(x \odot y) \odot(x \odot z)=\left(x y^{-1}\right) \odot\left(x z^{-1}\right)$,

$$
\begin{aligned}
& =\left(x y^{-1}\right)\left(x z^{-1}\right)^{-1}, \\
& =\left(x y^{-1}\right)\left(z x^{-1}\right), \\
& =x\left(y^{-1} z\right) x^{-1}, \\
& =\left(x\left(z^{-1} y\right)^{-1}\right) x^{-1}, \\
& =\left(x\left(z^{-1} y\right)^{-1}\right) \odot x, \\
& =\left(x \odot\left(z^{-1} y\right)\right) \odot x, \\
& =\left(x \odot\left(z^{-1} \odot y^{-1}\right)\right) \odot x, \\
& =(x \odot((e \odot z) \odot(e \odot y))) \odot x .
\end{aligned}
$$

(2) $x \odot(x \odot y)=x \odot\left(x y^{-1}\right)$

$$
\begin{aligned}
& =x\left(x y^{-1}\right)^{-1}, \\
& =(x y) x^{-1}, \\
& =(x y) \odot x, \\
& =\left(x \odot y^{-1}\right) \odot x, \\
& =(x \odot(e \odot y)) \odot x,
\end{aligned}
$$

(3) $x \odot x .=x \cdot x^{-1}=1=e$.

(4) $x \odot e=x \cdot e^{-1}=x \cdot e=x \cdot 1=x$.

(5) $e \odot x=e \cdot x^{-1}=1 \cdot x^{-1}=x^{-1}$.

Jadi terbukti bahwa $(\mathbb{R}-\{0\}, \cdot, \odot, 1)$ adalah suatu $\mathrm{K}$-Aljabar.

Definisi 2.3. [1] Suatu K-Aljabar $(G, *, \odot, e)$ adalah $K$-Aljabar abelian jika untuk setiap $x, g \in G$ berlaku $g \odot(e \odot x)=x \odot(e \odot g)$.

Proposisi 2.4. $(G, *, \odot, e)$ adalah suatu $K$-Aljabar abelian jika dan hanya jika $(G, *)$ adalah grup abelian.

Bukti. Misalkan $(G, *, \odot, e)$ adalah suatu K-Aljabar abelian, akibatnya untuk setiap $x, g \in G$, berlaku 


$$
g \odot(e \odot x)=x \odot(e \odot g),
$$

akibatnya,

$$
\begin{aligned}
g \odot\left(e x^{-1}\right) & =x \odot\left(e g^{-1}\right), \\
g \odot\left(x^{-1}\right) & =x \odot\left(g^{-1}\right), \\
g\left(\left(x^{-1}\right)^{-1}\right) & =x\left(\left(g^{-1}\right)^{-1}\right), \\
g x & =x g .
\end{aligned}
$$

Karena $g * x=x * g$, untuk setiap $x, g \in G$ maka $(G, *)$ adalah grup abelian.

Selanjutnya misalkan $(G, *)$ adalah grup abelian. Akan ditunjukkan $(G, *, \odot, e)$ adalah suatu K-Aljabar abelian. Karena $(G, *)$ adalah grup abelian, akibatnya untuk setiap $x, g \in G$, berlaku $g x=x g$.

Perhatikan bahwa:

$$
\begin{aligned}
g \odot(e \odot x) & =g \odot\left(x^{-1}\right), \\
& =g\left(x^{-1}\right)^{-1}, \\
& =g x, \\
& =x g, \\
& =x\left(g^{-1}\right)^{-1}, \\
& =x \odot\left(g^{-1}\right), \\
& =x \odot(e \odot g) .
\end{aligned}
$$

Karena $g \odot(e \odot x)=x \odot(e \odot g)$, untuk setiap $x, g \in G$, maka terbukti $(G, *, \odot, e)$ adalah suatu K-Aljabar abelian.

Proposisi 2.5. [1] Misalkan $(G, *, \odot, e)$ suatu K-Aljabar maka berlaku:

(1) $(x \odot y) \odot(u \odot v)=(x \odot(e \odot v) \odot(e \odot y)) \odot u$,

(2) $(x \odot y) \odot z=x \odot(z \odot(e \odot y))$,

(3) $e \odot(e \odot x)=x$,

(4) $e \odot(x \odot y)=y \odot x$

(5) $x \odot y=e$ jika dan hanya jika $x=y$.

Bukti. Misalkan $(G, *, \odot, e)$ merupakan suatu K-Aljabar. Ambil sebarang unsur $x, y, z, u, v \in G$ dan misalkan e unsur identitas di $\mathrm{G}$, maka untuk setiap $x, y, z, u, v \in$ $G$ berlaku :

(1) $(x \odot y) \odot(u \odot v)=\left(x y^{-1}\right) \odot\left(u v^{-1}\right)$,

$$
\begin{aligned}
& =\left(x y^{-1}\right)\left(u v^{-1}\right)^{-1}, \\
& =\left(x y^{-1}\right)\left(v u^{-1}\right), \\
& =\left(x y^{-1} v\right) u^{-1}, \\
& =x\left(y^{-1} v\right) u^{-1}, \\
& =x\left(v^{-1} y\right)^{-1} u^{-1}, \\
& =\left(x\left(v^{-1} y\right)^{-1}\right) u^{-1}, \\
& =\left(x\left(v^{-1} y\right)^{-1}\right) \odot u, \\
& =\left(x \odot\left(v^{-1} y\right)\right) \odot u, \\
& =\left(x \odot\left(v^{-1} \odot y^{-1}\right)\right) \odot u, \\
& =(x \odot(e \odot v) \odot(e \odot y)) \odot u .
\end{aligned}
$$


(2) $(x \odot y) \odot z=\left(x y^{-1}\right) \odot z$,

$$
\begin{aligned}
& =\left(x y^{-1}\right) z^{-1}, \\
& =x\left(y^{-1} z^{-1}\right), \\
& =x(z y)^{-1}, \\
& =x \odot(z y), \\
& =x \odot\left(z \odot y^{-1}\right), \\
& =x \odot(z \odot(e \odot y)) .
\end{aligned}
$$

(3) $e \odot(e \odot x)=e \odot\left(e x^{-1}\right)$,

$$
\begin{aligned}
& =e \odot x^{-1}, \\
& =e x,
\end{aligned}
$$$$
=x \text {. }
$$

(4) $e \odot(x \odot y)=e \odot\left(x y^{-1}\right)$,

$$
\begin{aligned}
& =e\left(x y^{-1}\right)^{-1}, \\
& =\left(x y^{-1}\right)^{-1},
\end{aligned}
$$$$
=y x^{-1} \text {, }
$$$$
=y \odot x \text {. }
$$

(5) Perhatikan bahwa:

$$
\begin{aligned}
(\Rightarrow) x \odot y=e & \Rightarrow x y^{-1}=e, \\
& \Rightarrow\left(x y^{-1}\right) y=e y, \\
& \Rightarrow x\left(y^{-1} y\right)=y, \\
& \Rightarrow x e=y, \\
& \Rightarrow x=y . \\
(\Leftarrow) x=y & \Rightarrow x y^{-1}=y y^{-1}, \\
\Rightarrow & (x \odot y)=e .
\end{aligned}
$$

\subsection{K-Subaljabar}

Definisi 2.6. [1] Misalkan $(G, *, \odot, e)$ suatu K-Aljabar. Suatu himpunan tak kosong $H$, dengan $H \subseteq G$ disebut $K$-Subaljabar jika:

(1) $e \in H$,

(2) $h_{1} \odot h_{2} \in H$, untuk setiap $h_{1}, h_{2} \in H$.

Proposisi 2.7. [1] Misalkan $(G, *, \odot, e)$ suatu K-Aljabar dan $g \in G$. Jika H suatu subgrup dari $G$, maka $H_{g^{2}}=\{g \odot(g \odot x) \mid x \in G\}$ adalah suatu K-Subaljabar dari $(G, *, \odot, e)$.

\section{Bukti.}

(1) Akan ditunjukkan $e \in H_{g^{2}}$. Karena $(G, *, \odot, e)$ suatu K-Aljabar maka $e$ unsur identitas di $G$. Perhatikan bahwa:

$$
\begin{aligned}
e & =e e \\
& =\left(g g^{-1}\right) e, \\
& =g\left(g^{-1} e\right) \\
& =g\left(e^{-1} g\right)^{-1}, \\
& =g(e g)^{-1}
\end{aligned}
$$




$$
\begin{aligned}
& =g \odot(e g), \\
& =g \odot(g e), \\
& =g \odot\left(g e^{-1}\right), \\
& =g \odot(g \odot e) \in H_{g^{2}} .
\end{aligned}
$$

Jadi $e \in H_{g^{2}}$.

(2) Ambil sebarang unsur $h_{1}, h_{2} \in H_{g^{2}}$. Akan ditunjukkan $h_{1} \odot h_{2} \in H_{g^{2}}$. Dari $h_{1}, h_{2} \in H_{g^{2}}$ diperoleh $h_{1}=g \odot(g \odot u)$ dan $h_{2}=g \odot(g \odot v)$, untuk suatu $u, v \in G$, akibatnya:

Jadi $h_{1} \odot h_{2} \in H_{g^{2}}$.

$$
\begin{aligned}
h_{1} \odot h_{2} & =(g \odot(g \odot u)) \odot(g \odot(g \odot v)), \\
& =\left(g\left(g u^{-1}\right)^{-1}\right) \odot\left(g\left(g v^{-1}\right)^{-1}\right), \\
& =\left(g\left(u g^{-1}\right)\right)\left(g\left(g v^{-1}\right)^{-1}\right)^{-1}, \\
& =\left(g\left(u g^{-1}\right)\right)\left(\left(g v^{-1}\right) g^{-1}\right), \\
& =g u\left(g^{-1} g\right) v^{-1} g^{-1}, \\
& =g u v^{-1} g^{-1}, \\
& =g\left(\left(u v^{-1}\right) g^{-1}\right), \\
& =g\left(g\left(u v^{-1}\right)^{-1}\right)^{-1} \\
& =g \odot\left(g\left(u v^{-1}\right)^{-1}\right), \\
& =g \odot(g \odot(u \odot v)) \in H_{g^{2}} .
\end{aligned}
$$

Jadi terbukti bahwa $H_{g^{2}}=\{g \odot(g \odot x) \mid x \in G\}$ adalah suatu K-Subaljabar dari $(G, *, \odot, e)$.

\subsection{K-Homomorfisma}

Definisi 2.8. [1] Misalkan $K=(G, *, \odot, e)$ dan $\bar{K}=(\bar{G}, \bar{*}, \odot, \bar{e})$ merupakan $K$ Aljabar. Suatu pemetaan $\varphi$ dari $K$ ke $\bar{K}$, disebut $K$-Homomorfisma jika untuk setiap $x, y \in K$ berlaku $\varphi(x \odot y)=\varphi(x) \odot \varphi(y)$, dimana $\varphi(x), \varphi(y) \in \bar{K}$.

Contoh 2.9. Misalkan $(G, *, \odot, e)$ merupakan suatu K-Aljabar dan $g \in G$. Selanjutnya didefinisikan himpunan $H=\{g \odot(g \odot x) \mid x \in G\}$. Misalkan $(H, *, \odot, e)$ merupakan suatu K-Aljabar dan $\varphi:(G, *, \odot, e) \rightarrow(H, *, \odot, e)$ suatu pemetaan dengan $\varphi(x)=g \odot(g \odot x), \forall x \in G$. Akan ditunjukkan $\varphi:(G, *, \odot, e) \rightarrow(H, *, \odot, e)$ merupakan suatu K-Homomorfisma. Ambil $a, b \in G$, karena $\mathrm{G}$ suatu grup maka $a \odot b=a b^{-1} \in G$. Akibatnya:

$$
\begin{aligned}
\varphi(a \odot b) & =g \odot(g \odot(a \odot b)), \\
& =g \odot\left(g \odot\left(a b^{-1}\right)\right), \\
& =g \odot\left(g\left(a b^{-1}\right)^{-1}\right), \\
& =g\left(g\left(a b^{-1}\right)^{-1}\right)^{-1}, \\
& =g\left(\left(a b^{-1}\right) g^{-1}\right), \\
& =g a b^{-1} g^{-1}, \\
& =g a e b^{-1} g^{-1} \\
& =g a\left(g^{-1} g\right) b^{-1} g^{-1}, \\
& =\left(g a g^{-1}\right)\left(g b^{-1} g^{-1}\right), \\
& =\left(g a g^{-1}\right)\left(g(g b)^{-1}\right), \\
& =\left(g a g^{-1}\right)\left((g b) g^{-1}\right)^{-1},
\end{aligned}
$$




$$
\begin{aligned}
& =\left(g a g^{-1}\right) \odot\left((g b) g^{-1}\right), \\
& =\left(g\left(a g^{-1}\right)\right) \odot\left(g\left(b g^{-1}\right)\right), \\
& =\left(g\left(g a^{-1}\right)^{-1}\right) \odot\left(g\left(g b^{-1}\right)^{-1}\right), \\
& =\left(g \odot\left(g a^{-1}\right)\right) \odot\left(g \odot\left(g b^{-1}\right)\right), \\
& =(g \odot(g \odot a)) \odot(g \odot(g \odot b)), \\
& =\varphi(a) \odot \varphi(b) .
\end{aligned}
$$

Karena $\varphi(a \odot b)=\varphi(a) \odot \varphi(b)$, maka terbukti pemetaan $\varphi:(G, \odot) \rightarrow(H, \odot)$ merupakan suatu K-Homomorfisma.

Pada grup dikenal konsep monomorfisma, epimorfisma dan isomorfisma. Seperti pada grup, pada K-Aljabar juga dikenal konsep K-Monomorfisma, K-Epimorfisma dan K-Isomorfisma yang akan diberikan berikut ini.

Misalkan $(G, *, \odot, e)$ dan $(\bar{G}, \bar{*}, \bar{\odot}, \bar{e})$ merupakan K-Aljabar, dan pemetaan $\varphi$ : $G \rightarrow \bar{G}$ adalah K-Homomorfisma, maka:

(1) Pemetaan $\varphi: G \rightarrow \bar{G}$ disebut K-Monomorfisma, jika $\varphi$ suatu pemetaan satusatu (injektif), yaitu untuk setiap $x, y \in G$ dengan $\varphi(x)=\varphi(y)$, maka $x=y$.

(2) Pemetaan $\varphi: G \rightarrow \bar{G}$ disebut K-Epimorfisma, jika $\varphi$ suatu pemetaan pada (surjektif), yaitu untuk setiap $y \in \bar{G}$, maka terdapat $x \in G$ sehingga $\varphi(x)=y$.

(3) Pemetaan $\varphi: G \rightarrow \bar{G}$ disebut K-Isomorfisma, jika $\varphi$ suatu pemetaan bijektif (injektif dan surjektif)[1].

Definisi 2.10. [1] Misalkan $K=(G, *, \odot, e)$ dan $\bar{K}=(\bar{G}, \bar{*}, \bar{\odot}, \bar{e})$ merupakan dua $K$-Aljabar, maka himpunan semua $K$-Homomorfisma dari $K$ ke $\bar{K}$ dinotasikan,

$$
\operatorname{Hom}(K, \bar{K}) \text {. }
$$

Proposisi 2.11. [1] Misalkan $K=(G, *, \odot, e)$ dan $\bar{K}=(\bar{G}, \bar{*}, \odot, \bar{e})$ merupakan dua $K$-Aljabar dan $\varphi \in \operatorname{Hom}(K, \bar{K})$, maka untuk setiap $x, y \in K$ dan $\varphi(x), \varphi(y) \in \bar{K}$, maka berlaku:

(1) $\varphi(e)=\bar{e}$, untuk suatu e unsur identitas di $K$ dan $\bar{e}$ unsur identitas di $\bar{K}$,

(2) $\varphi\left(x^{-1}\right)=\varphi(x)^{-1}$,

(3) $\varphi(e \odot x)=\bar{e} \odot \varphi(x)$,

(4) $\varphi(x \odot y)=\bar{e}$, jika dan hanya jika $\varphi(x)=\varphi(y)$,

(5) Jika $H$ adalah $K$-Subaljabar dari $K$, maka $\varphi(H)$ adalah $K$-Subaljabar dari $\bar{K}$.

Bukti. Misalkan $K=(G, *, \odot, e)$ dan $\bar{K}=(\bar{G}, \bar{*}, \bar{\odot}, \bar{e})$ merupakan dua K-Aljabar dan $\varphi \in \operatorname{Hom}(K, \bar{K})$. Misalkan $e$ unsur identitas di $K$ dan $\bar{e}$ unsur identitas di $\bar{K}$. Ambil $x, y \in K$, karena $\varphi \in \operatorname{Hom}(K, \bar{K})$ maka $\varphi(x), \varphi(y) \in \bar{K}$ dan berlaku $\varphi(x \odot y)=\varphi(x) \bar{\odot} \varphi(y)$.

Perhatikan bahwa:

(1) $\varphi(e)=\varphi(x \odot x)$,

$$
\begin{aligned}
& =\varphi(x) \odot \varphi(x), \\
& =\varphi(x) \bar{*} \varphi(x)^{-1},
\end{aligned}
$$

$$
=\bar{e} \text {. }
$$


(2) $\varphi\left(x^{-1}\right)=\varphi(e \odot x)$,

$$
\begin{aligned}
& =\varphi(e) \odot \varphi(x), \\
& =\varphi(e) \bar{*} \varphi(x)^{-1}, \\
& =\bar{e} \bar{*} \varphi(x)^{-1}, \\
& =\varphi(x)^{-1} .
\end{aligned}
$$

(3) $\varphi(e \odot x)=\varphi(e) \odot \varphi(x)$,

$$
=\bar{e} \odot \varphi(x) \text {. }
$$

(4) $(\Rightarrow) \varphi(x \odot y)=\bar{e} \Rightarrow \varphi(x) \odot \varphi(y)=\bar{e}$,

$$
\begin{aligned}
& \varphi(x) \bar{*} \varphi(y)^{-1}=\bar{e}, \\
& \varphi(x) \bar{*} \varphi(y)^{-1} \bar{*} \varphi(y)=\bar{e} \bar{*} \varphi(y), \\
& \varphi(x) \bar{*}\left(\varphi(y)^{-1} \bar{*} \varphi(y)\right)=\varphi(y), \\
& \varphi(x) \bar{*} \bar{e}=\varphi(y), \\
& \varphi(x)=\varphi(y) . \\
(\Leftarrow) \varphi(x)=\varphi(y) \Rightarrow & \varphi(x) \odot \varphi(y)=\varphi(y) \odot \varphi(y), \\
& \varphi(x \odot y)=\varphi(y \odot y), \\
& \varphi(x \odot y)=\varphi(e), \\
& \varphi(x \odot y)=\bar{e},
\end{aligned}
$$

(5) Misalkan $H$ merupakan K-Subaljabar dari $K$. Akan ditunjukkan bahwa $\varphi(H)$ adalah K-subaljabar dari $\bar{K}$ sebagai berikut:

(a) Karena $H$ merupakan K-Subaljabar dari $K$ dan $e$ suatu unsur identitas di $K$, maka $e \in H$. Akibatnya $\varphi(e)=\bar{e} \in \varphi(H)$, untuk suatu $\bar{e}$ unsur identitas di $\bar{K}$,

(b) Selanjutnya ambil $u, v \in \varphi(H)$, yaitu $u=\varphi\left(h_{1}\right)$ dan $v=\varphi\left(h_{2}\right)$, untuk suatu $h_{1}, h_{2} \in H$. Akan ditunjukkan $u \odot v \in \varphi(H)$.

Perhatikan bahwa:

$$
\begin{aligned}
u \bar{\odot} v & =\varphi\left(h_{1}\right) \odot \varphi\left(h_{2}\right), \\
& =\varphi\left(h_{1} \odot h_{2}\right), \\
& =\varphi\left(h_{1} * h_{2}^{-1}\right), \\
& =\varphi\left(h_{3}\right), \text { untuk suatu } h_{3}=h_{1} * h_{2}^{-1} \in H .
\end{aligned}
$$

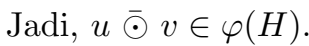

Karena $\bar{e} \in \varphi(H)$, untuk suatu $\bar{e}$ unsur identitas di $\bar{K}$ dan untuk sebarang unsur $u, v \in \varphi(H)$ berlaku $u \odot v \in \varphi(H)$, maka $\varphi(H)$ adalah suatu K-Subaljabar dari $\bar{K}$.

\section{Kesimpulan}

K-Aljabar $(G, *, \odot, e)$ dengan $e$ adalah suatu unsur identitas di $G$ dibangun dari suatu grup dengan menggunakan operasi biner $\odot$ pada grup $(G, *)$ yang didefinisikan sebagai $x \odot y=x * y^{-1}$, untuk setiap $x, y \in G$, sedemikian sehingga memenuhi aksioma-aksioma tertentu.

Dari suatu K-Aljabar dapat dibentuk satu atau lebih himpunan bagian yang juga memiliki sifat K-Aljabar terhadap operasi biner yang sama yang kemudian himpunan bagian tersebut dapat dinamakan K-Subaljabar. Sebagaimana halnya pada grup yang terdapat konsep homomorfisma grup, pada K-Aljabar juga terdapat konsep homomorfisma yang dinamakan K-Homomorfisma. 
100 Meza Aprilisa dkk

\section{Daftar Pustaka}

[1] Dar, K. H. dan Akram, M. 2006. On K-Homomorphisms of K-algebras. International Mathematical Forum. 46 : 2283 - 2288.

[2] Fraleigh, J. B. 1994. A First Course in Abstract Algebra. United States. AddisonWesley Publishing Company Inc.

[3] Herstein, I.N. 1996. Abstract Algebra, Third Edition. Prentice-Hall, Inc, United States of America.

[4] Hungerford, T.W. 1974. Algebra. Springer-Verlag New York, Inc. New York. 